\title{
A
}

\section{Suppression of Gate Oxide Degradation for MOS Devices Using Deuterium Ion Implantation Method}

\author{
Jae-Sung Lee Le $^{+}$ \\ Department of Information and Communication Engineering, Uiduk University, Gyeongju 780-713, Korea
}

Received May 7, 2012; Revised June 12, 2012; Accepted June 12, 2012

\begin{abstract}
This paper introduces a new method regarding deuterium incorporation in the gate dielectric including deuterium implantation and post-annealing at the back-end-of-the process line. The control device and the deuterium furnaceannealed device were also prepared for comparison with the implanted device. It was observed that deuterium implantation at a light dose of $1 \times 10^{12}-1 \times 10^{14} / \mathrm{cm}^{2}$ at $30 \mathrm{keV}$ reduced hot-carrier injection (HCI) degradation and negative bias temperature instability (NBTI) within our device structure due to the reduction in oxide charge and interface trap. Deuterium implantation provides a possible solution to enhance the bulk and interface reliabilities of the gate oxide under the electrical stress.
\end{abstract}

Keywords: Deuterium, Ion implantation, Gate oxide, Reliability

\section{INTRODUCTION}

The reliability characteristics of ultrathin $\mathrm{SiO}_{2}$ film, which serves as the gate dielectric in silicon metal-oxide-semiconductor (MOS) devices, are one of the important issues to realize highly integrated devices. Deuterium incorporation compared with that of hydrogen at the $\mathrm{Si} / \mathrm{SiO}_{2}$ area is known to improve device reliability [1]. The deuterium isotope effect is based on a much slower desorption rate of deuterium atoms from the $\mathrm{Si} / \mathrm{SiO}_{2}$ interface. Deuterium has been incorporated into MOS devices by substituting deuterium gas for forming gas during post-metallization annealing under atmospheric pressure. This reduces hot-carrier injection (HCI) degradation in the MOS transistor by passivating the interface states at the $\mathrm{Si} / \mathrm{SiO}_{2}$ interface. However, there is no consensus on the influence of deuterium incorporation on other degradations, such as negative bias temperature instability (NBTI) and stress-induced leakage current (SILC) [2,3].

For the integration of deuterium annealing into the main-

${ }^{\dagger}$ Author to whom all correspondence should be addressed:

E-mail: jaesung@uu.ac.kr

Copyright $\odot 2012$ KIEEME. All rights reserved.

This is an open-access article distributed under the terms of the Creative Commons Attribution Non-Commercial
License (http://creativecommons.org/licenses/by-nc/3.0) which permits unrestricted noncommercial use,

Listribution, and reproduction in any medium, provided the original work is properly cited.
ditiction stream with regards to semiconductor manufacturing, significant challenges still remain. When complementary-metal-oxidesemiconductor (CMOS) technologies incorporate multiple metals and dielectrics the improvement due to deuterium annealing was reduced further [4]. Deuterium diffusion takes place primarily through the silicon oxide $\left(\mathrm{SiO}_{2}\right)$ in the MOS system due to the limited permeability of bulk $\mathrm{Si}$, metal and even polysilicon to deuterium [5]. Deuterium implantation, on the other hand, can provide a spatially uniform distribution of deuterium throughout the gate oxide area [6]. Recently, deuterium implantation being carried out prior to the growth of gate oxide was reported [7]. However, it increased the silicon surface damage and the thickness of gate oxide compared with that of the control. In addition, the impact of deuterium implantation on device reliability, such as HCI and NBTI, has not yet been reported. The quality of gate oxide benefits from a deuterium isotope effect only when the deuterium is incorporated at the end of the process, in order to avoid desorption of the deuterium during hightemperature processing steps.

In this work, we report the reliability characteristics of MOSFET, including HCI and NBTI, when deuterium was incorporated in the gate oxide region by ion implantation after the first metallization. The implantation conditions for deuterium ion were calculated in advance through computer simulation (SRIM tool). Deuterium implantation is provided at low energy, and then subsequent post-annealing is achieved for the deuterium diffusion 
(a)

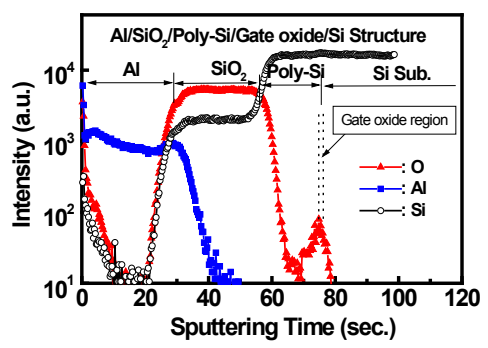

(b)

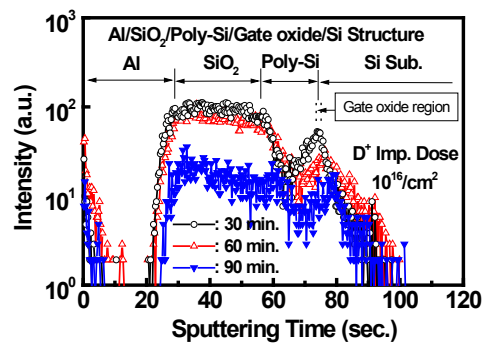

Fig. 1. SIMS concentration profiles for (a) $\mathrm{Al}, \mathrm{Si}$, and $\mathrm{O}$ and (b) deuterium, $\mathrm{D}$, in the deuterium implanted $\mathrm{Al} / \mathrm{SiO}_{2} /$ poly-Si/gate oxide/Si substrate structure. The profile in Fig.1 (a) was achieved after postannealing for $30 \mathrm{~min}$. in $\mathrm{N}_{2}$ ambience and Fig. 1(b) represents variation of deuterium profile depending on annealing time.

into the gate oxide area.

\section{EXPERIMENTS}

MOS devices, $\mathrm{p}$ - and n-MOSFET, were fabricated using standard $0.18 \mu \mathrm{m}$ CMOS technology for channel lengths down to $0.15 \mu \mathrm{m}$. The effective oxide thickness was in a range of $3 \mathrm{~nm}$. The gate oxide film was produced with a conventional furnace in NO gas ambient. The deuterium implantation was performed at the back-end of the process line. The total thickness from the silicon surface to the top of the first metal was approximately 700 $\mathrm{nm}$, including aluminum, silicon dioxide, and polysilicon layers. Post-annealing after implantation was achieved at $400^{\circ} \mathrm{C}$ in $\mathrm{N}_{2}$ ambient for all devices in order to diffuse the injected ions and remove damages due to the implant process. The normal processed device (hydrogen furnace-annealed device) and the deuterium furnace-annealed device were also prepared to compare with the implant processed device.

\section{RESULTS}

Deuterium was implanted into our processed MOSFET with implantation energy of $30 \mathrm{keV}$ and with a dose range of $1 \times 10^{12}$ $1 \times 10^{14}$ atoms $/ \mathrm{cm}^{2}$. We found that the implantation with higher energy and more doses made the reliability worse and that with lower energy and fewer doses provided no deuterium effect within our device. The material stack of MOSFET in our wafer was composed of $\mathrm{SiO}_{2}$ [300 nm] / poly-Si [250 nm] / gate oxide [3 $\mathrm{nm}]$ / Si-substrate.

Secondary ion mass spectroscopy (SIMS) measurement for $\mathrm{Al} / \mathrm{SiO}_{2} /$ poly-Si/gate oxide/Si substrate which was implanted by deuterium on the top $\mathrm{Al}$ with $85 \mathrm{keV}$ energy and $10^{16} / \mathrm{cm}^{2}$ dose is shown in Fig. 1. The aluminium layer covered the dielectric layers which solved the charging problem during ion sputtering for the depth profile. The implantation condition was chosen to make the peak of ion range localized in the middle of the polysilicon layer. From Fig. 1(a), each layer and its thickness above the

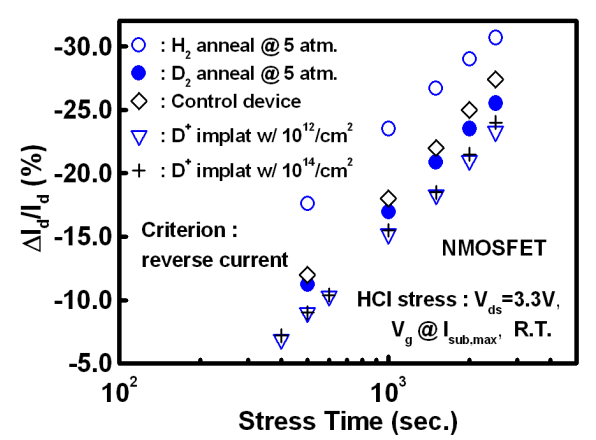

Fig. 2. Stress time dependence of $\Delta I_{d} / I_{d}$ for deuterated n-MOSFET's stressed in the $\mathrm{HCI}$ condition, biased $\mathrm{V}_{\mathrm{ds}}$ was fixed to $3.3 \mathrm{~V}$ and $\mathrm{V}_{\mathrm{g}}$ was chosen as the value that the maximum substrate current flowed. The control device and the hydrogen-annealed device were also shown to compare with deuterium-annealed and implanted devices. The device annealed at $1 \mathrm{~atm}$. Deuterium ambience was not shown here, but its degradation curve could be positioned between curves of the control device and the 5 atm.- $\mathrm{D}_{2}$-annealed device.

Si substrate were estimated and the thin gate oxide is assumed to be between the polysilicon and the Si substrate. Fig. 1(b) shows the depth profiles of deuterium depending on post-annealing time. The deuterium concentrations at the two $\mathrm{SiO}_{2}$ regions, including the gate oxide area, are higher than that in other layers when annealing time was $30 \mathrm{~min}$. The implanted ions in the polysilicon layer diffuse toward the gate oxide area when postannealing is processing due to the chemical potential difference between $\mathrm{SiO}_{2}$ and $\mathrm{Si}$ [8]. Consequently, deuterium atoms are profiled in the $3 \mathrm{~nm}$-thick gate oxides. The annealing time of $30 \mathrm{~min}$. was observed to be an optimum time for post-annealing in our device structure.

Passivation of dangling bonds at the $\mathrm{Si} / \mathrm{SiO}_{2}$ interface can be evaluated by estimating the variation of drain current after HCI stress. Fig. 2 shows the decrease of reverse saturation drain current for devices processed with different deuterium incorporation methods as a function of HCI stress time. The results for the control device and the high-pressure hydrogen-annealed device are represented in order to compare them to that of the deuterium-implanted device. Deuterium annealing shows the suppression of HCI degradation that was already demonstrated by Hess [9]. He demonstrated the deuterium effect under the atmosphere ( 1 atm.) ambience of deuterium gas. We also found the same result, which was the suppression of drain current decrease under HCI stress. Therefore, the degradation curve for the 1 atm.- $\mathrm{D}_{2}$-annealed device could be positioned between that of the control device and that of the 5 atm.- $\mathrm{D}_{2}$-annealed device in Fig. 2. In the annealing process, the higher deuterium concentration is expected at the drain and source end of the channel due to the presence of sidewall spacers and a polysilicon layer above the channel area. That is, deuterium diffusion can have a localized effect. Deuterium-implanted devices have resulted in better suppression of degradation compared to annealed devices. The identical behavior regarding the current decrease was observed for $1 \times 10^{12}$ and $1 \times 10^{14}$ atoms $/ \mathrm{cm}^{2}$ implanted devices. In the implantation process, the depth at which concentration peak occurs was in the middle of the polysilicon area. Therefore, during subsequent $\mathrm{N}_{2}$ annealing deuterium has to diffuse to reach the interface along the channel. Only when deuterium with an optimum concentration is introduced will the oxide interface be more stable. When deuterium was implanted with more than $1 \times 10^{14}$ atoms $/ \mathrm{cm}^{2}$ HCI degradation was increased further even more so than the control device. In Fig. 3, we also found an isotope effect in the high-pressure hydrogen and deuterium anneal- 


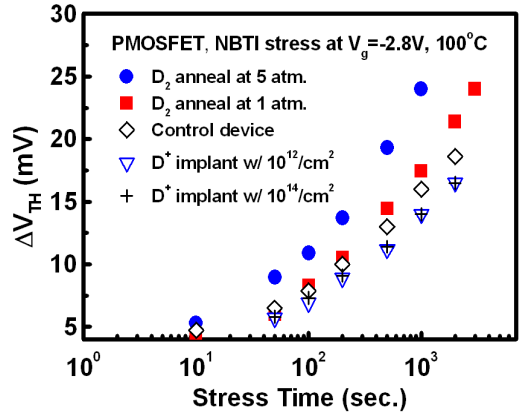

Fig. 3. Time dependence for the shift of threshold voltage $\left(\mathrm{V}_{\text {TH }}\right)$ for deuterated p-MOSFET's stressed at NBTI condition, $\mathrm{V}_{\mathrm{g}}=-2.8 \mathrm{~V}$, and $\mathrm{V}_{\mathrm{ds}}=\mathrm{V}_{\mathrm{sub}}=0 \mathrm{~V}$ at $100^{\circ} \mathrm{C}$. Shift of $\mathrm{V}_{\mathrm{TH}}$ means the generation of positive charged atoms in the gate oxide.

ing processes.

Figure 3 shows the shift of threshold voltage $\left(\mathrm{V}_{\mathrm{TH}}\right)$ for deuterium-incorporated p-MOSFET's that were stressed by using NBTI condition. As for NBTI stress on MOSFET, both interfaceand bulk-reaction are possibly the reasons for the degradation. Therefore, NBTI degradation is commonly observed in the whole gate oxide area, which is in contrast with HCI degradation. In Fig. 3 , the shift of $\mathrm{V}_{\mathrm{TH}}$ is suppressed in the deuterium-implanted device compared to the control device, and no improvement of $\mathrm{V}_{\mathrm{TH}}$ instability was observed in the deuterium-annealed device. During the annealing process, we found that NBTI was accelerated in a high-pressure ambience, which implies that surplus deuterium concentration in the gate oxide created more extrinsic defect in the oxide bulk and in the oxide interface near the drain and source sides. With regards to the hydrogen process we found the generation of defect clearly. A significant shift of $\mathrm{V}_{\mathrm{TH}}$ was found in the high-pressure (5 atm.) hydrogen-annealed device under the same NBTI condition, which implies that oxide-fixed charge is more easily generated at the high-hydrogenated device. The generation of extrinsic defect related with deuterium annealing has been reported by our group [10]. In the implantation process, deuterium can be spread into the gate oxide bulk through the optimum dose and energy. The NBTI condition leads to uniform donor-like interface trap generation in gate oxide interface of $\mathrm{p}$ MOSFET and then injects channel holes into the gate oxide [11]. However, we have found that the effect of deuterium treatment was not shown at the gate oxide interface in p-MOSFET under the inversion bias mode [10]. Therefore, the suppression of NBTI degradation by deuterium implantation is attributed to the enhancement of gate oxide bulk quality.

Under the NBTI stress condition the channel holes can tunnel toward the thin gate oxide, and potentially gain the energy to break the $\mathrm{Si}-\mathrm{H}(\mathrm{D})$ bonds inside the gate oxide. Based on such a degradation scenario, the deuterium effect shown in Fig. 3 is due to the bulk-reaction, not the interface-reaction. For application in the CMOS process, deuterium annealing is a convenient process to improve the interface property of gate oxide. However, deuterium implantation has an advantage for enhancing the oxide bulk quality and the interface property concurrently by the optimum implantation condition.

The stress-induced leakage current (SILC) under FowlerNordheim (FN) stress can be used to estimate the trap creation in the bulk of gate oxide. Fig. 4 represents the variation of gate current for deuterium-implanted p-MOSFETs, and its value was compared with that of the hydrogen-implanted device. The ion range of hydrogen implantation was adjusted to the same level of deuterium implantation. The current was measured at the gate terminal while the gate voltage was being swept from 0 to 3

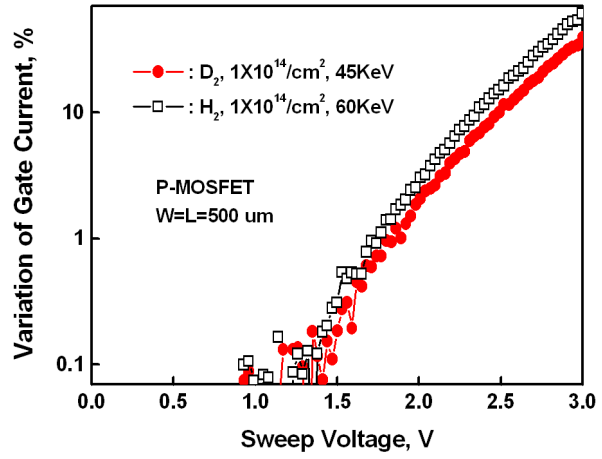

Fig. 4. Percentage variation of gate leakage current, between before and after a constant voltage stress $\left(V_{g}=-3.5 \mathrm{~V}\right)$, for $\mathrm{p}$-MOSFET's implanted with hydrogen and deuterium, respectively. The ion ranges for both atoms were in the same value.

$\mathrm{V}$ after finishing the constant voltage stress $\left(\mathrm{V}_{\mathrm{g}}=-3.5 \mathrm{~V}\right)$. The device with deuterium implantation showed a lower gate leakage current for the entire range of sweeping voltages. The generation of stress-related bulk-oxide traps, which is a cause for leakage current, is suppressed through the incorporation of deuterium. We can then infer that the isotope effect is valid in our suggested implantation method. Therefore, deuterium implantation could be used to improve reliability, if a suitable implant condition is obtained.

\section{CONCLUSIONS}

We have demonstrated that the incorporation of deuterium in the gate oxide using ion implantation in the back-end process is an effective means to improve oxide quality and may be a viable alternative to deuterium furnace annealing. Isotope effect between hydrogen-implanted and deuterium-implanted devices was also demonstrated. Deuterium implantation brings about a reliability enhancement by suppressing the HCI and NBTI degradations. The obtained results show that the oxide bulk and the oxide interface benefit from the implantation of deuterium by optimizing the ion range, the dose, the energy, and the following $\mathrm{N}_{2}$ annealing by adjusting annealing time. The deuterium isotope effect in MOS device appears only when oxide degradation is precipitated by energetic "hot" carriers. It means that the deuterium and the hydrogen bonds within the gate oxide interface are desorbed at the same rate by non-energetic channel carriers. Finally, we demonstrated the reliability enhancement for the deuterium- implanted device by explaining the interface reaction in HCI stress and the bulk reaction in NBTI stress, respectively.

\section{ACKNOWLEDGMENTS}

This work was supported by the Proton Engineering Frontier Project (PEFP) funded by the Korean Government and the Uiduk University Research Grant, 2011.

\section{REFERENCES}

[1] J. W. Lyding, K. Hess, and I. C. Kizilyalli, Appl. Phys. Lett., 68, 2526 (1996) [DOI: 10.1063/1.116172].

[2] C. H. Liu, M.T. Lee, C. Lin, J. Chen, K. Schruefer, J. Brighten, N. Rovedo, T. B. Hook, M. V. Khare, S.-F. Huang, C. Wann, T. 
C. Chen, T. H. Ning, IEEE IEDM 2001 P. 861 [DOI: 10.1109/ IEDM.2001.979649].

[3] J. Wu, E. Rosenbaum, E. MacDonald, B. E. Li, B. Tracy, and P. Fang, IEEE Int. Reliability Physics Symp. 2000 p. 27 [DOI: 10.1109/RELPHY.2000.843887].

[4] J. Lee, Y. Epstein, A. C. Berti, J. Huber, K. Hess, J. W. Lyding, IEEE Trans. Electron Devices 46, 1812 (1999) [DOI: 10.1109/16.777177 ].

[5] W. F. Clark, T. G. Ference, T. B. Hook, K. M. Watson, S. W. Mitti, J .S. Burnham, IEEE Electron Device Lett. 20, 48 (1999) [DOI : $10.1109 / 55.737570$ ]
[6] K. C. Harvey, U.S. Patent 6017 806, Jan. 25, (2000).

[7] T. Kundu, D. Misra, IEEE Trans. Device and Material Reliability 6, 288 (2006) [DOI: 10.1109/TDMR.2006.876586].

[8] H. Park and C. R. Helms, J. Electrochem. Soc., 139, 2042 (1992) [DOI: 10.1149/1.2221171].

[9] K. Hess, I. C. Kizilyalli, J. W. Lyding, IEEE Trans. Electron Devices 45, 406 (1998) [DOI: 10.1109/16.658674].

[10] J. S. Lee, J. W. Lyding, K. Hess, IEEE Int. Reliability Phys. Symp. 2004 p. 685 [DOI: 10.1109/RELPHY.2004.1315451].

[11] D. K. Schroder, J. A. Babcock, J. Appl. Phys. 94, 1 (2003) [DOI: $10.1063 / 1.1567461]$. 